Sharif University of Technology
Scientia Iranica
Transactions E: Industrial Engineering
wCIENTIA

\title{
A two-echelon inventory model with perishable items and lost sales
}

\author{
A. Mahmoodi*, A. Haji and R. Haji \\ Department of Industrial Engineering, Sharif University of Technology, Tehran, Iran. \\ Received 4 May 2014; received in revised form 7 May 2015; accepted 6 September 2015
}

\section{KEYWORDS \\ Inventory; \\ Perishable items; \\ Base stock policy; \\ Lost sales; \\ Two-echelon.}

\begin{abstract}
This article deals with a single perishable item, continuous-review, two-echelon serial inventory system consisting of a warehouse and a retailer. Customer demands at the retailer are assumed to be Poisson. All items have a fixed shelf life and start aging on their arrival at the retailer. The demand that cannot be met immediately at the retailer is lost. All transportation times are fixed. If there is any stock in the warehouse, the lead time for the retailer would be the transportation time from the warehouse. Otherwise, the retailer orders are met with a delay. In this article, using an approximate technique, we first present a heuristic for finding cost-effective base stock policy and then develop a simulation-based neighborhood search procedure to modify the quality of the solution. Also, a numerical experiment is carried out to evaluate effectiveness and accuracy of the procedures. The results reveal that the approximate model performs rather well.
\end{abstract}

(C) 2016 Sharif University of Technology. All rights reserved.

\section{Introduction}

Most conventional inventory models are presented to deal with nonperishable items. However, perishable inventories are the major concern in many industrial sectors. Foodstuffs, pharmaceuticals, blood and its derivatives, batteries, and photographic films are a few examples of perishable inventories. By disregarding the finite lifetime of perishable items, the resulting model may give inaccurate results. Therefore, lifetime of the item has to be taken into consideration to develop a cost-effective inventory model.

In this article, a single perishable item, continuous-review, two-echelon serial inventory system consisting of a warehouse and a retailer is considered. The retailer faces Poisson demand and replenishes its stock from the warehouse. The warehouse, in turn, replenishes its stock through an external supplier. The transportation time from the warehouse to the retailer

\footnotetext{
*. Corresponding author. Tel.: +982166165704;

Fax: +9821 66022702

E-mail address: a_mahmoodi@ie.sharif.edu (A. Mahmoodi)
}

is fixed. If there is any stock in the warehouse, the lead time for the retailer would be the transportation time. Otherwise, the retailer orders are met with a delay. The lead time on a warehouse order is fixed. After joining the stock at retailer, an item has a constant shelf life, beyond which it is no longer usable. The demand that cannot be met immediately at the retailer is lost. The fixed ordering cost is negligible and both installations operate base stock policy to control their inventories.

To the best of our knowledge, the papers which study perishable items in multi-echelon inventory systems are very few. The optimal replenishment policies for a single perishable item, two-echelon inventory system were analyzed by Abdel-Malek and Ziegler [1]. They assumed deterministic demand for their inventory system. The problem of periodic review ordering and issuing policies in a two-echelon inventory system under demand uncertainty was considered by Fujiwara et al. [2]. However, they assumed unique lifetime for each echelon.

Furthermore, the effect of product perishability on total cost of the system in a two-echelon inventory system was investigated by Kanchanasuntorn and 
Techanitisawad [3]. They developed an approximate inventory model under periodic review policies assuming normal demand for retailers.

Moreover, Olsson [4] addressed a continuousreview, two-echelon serial inventory system with perishable items and developed an approximate technique for evaluation of base stock policies. Considering Poisson demand for the downstream location, he assumed that the unsatisfied demand was backordered. Finally, Mahmoodi and Haji [5] developed $(1, T)$ policy for a two-echelon inventory system with perishable-on-theshelf items.

From a modeling point of view, our model is related to single-echelon models with continuous review. Significant studies have been carried out for inventory control of single installations of perishable items. Schmidt and Nahmias [6] addressed a system with perishable items operating under $(S-1, S)$ policy with lost sales, Poisson demand, outdating costs, purchase costs, and per unit per period holding costs. Perry and Posner [7] generalized Schmidt and Nahmias [6] to allow for general types of customer impatience behavior. Olsson and Tydesjo [8] extended Schmidt and Nahmias [6] by allowing backorders. Mahmoodi et al. [9] considered an inventory system under Schmidt and Nahmias [6] assumptions but employed $(1, T)$ policy instead of $(S-1, S)$ policy. The interested reader can refer to [10] and [11], which are two comprehensive reviews focusing on inventory control of perishable items.

This paper presents an approach to deal with replenishment problem in a single perishable item, twoechelon inventory system under continuous review and lost sales. The considered inventory system in this paper is different from inventory systems studied in the literature. For example, our system is different from Abdel-Malek and Ziegler [1] in that we consider stochastic demand. Fujiwara et al. [2] and Kanchanasuntorn and Techanitisawad [3], unlike our continuous review, considered periodic review policies. Finally, although like Olsson [4] we consider continuous review, we assume lost sales.

For the items with infinite lifetime, our model is reduced to the one considered by Andersson and Melchiors [12]. However, the inclusion of perishability requires an entirely new analysis due to the fact that not only the perished items affect the demand of the warehouse but also the retailer should make tradeoff between the number of lost sales and the number of perished items.

Considering lost sales and perishable items, the demand process at the warehouse is not a Poisson process. We ignore this and approximate the demand process at the warehouse with a Poisson process. Then, the approximated long-run average total cost function of the inventory system is derived. Furthermore, two procedures are presented to obtain the optimal or nearoptimal solution. Our approach gives an approximate model which is quite simple and efficient from a computational point of view. Simulation results show that the performance is very good. Although a serial system is considered, the presented model could easily be extended to a distribution system with identical retailers.

An interesting application of our proposed model is to a situation where an operating equipment is subject to both failures and routine maintenance, which was introduced as an application of $(S-1, S)$ policy by Schmidt and Nahmias [6]. In particular, consider a facility with $S_{1}$ similar operating machines. Each machine has an engine which is subject to failure at random times. Any engine which has been in service for a fixed time $m$ without failure is also removed for maintenance. Each time an engine is removed for maintenance or repair, an order for replacement is made from a warehouse. The warehouse operates under $\left(S_{0}-1, S_{0}\right)$ policy. The transportation time from the warehouse to the facility is fixed and known. If there is any stock in the warehouse, the lead time for the facility would be the transportation time. Otherwise, the facility orders are met with a delay. The lead time on a warehouse order is fixed. The number of operating machines in the facility is as the inventory on hand in the retailer in the considered model. Our model can be used to determine not only the inventory position at the warehouse but also the fixed interval of routine maintenance (see [6] for more details).

The proceeding parts of this paper are organized as follows: in Section 2, the considered model is described. In Section 3, the cost function of the system is approximated. Then, in Section 4, two procedures are introduced to find the best policy for the model. Furthermore, a numerical analysis is carried out to evaluate accuracy and effectiveness of procedures in Section 5. Finally, the conclusion and future research are presented in Section 6.

\section{Model description}

We consider a single-item, two-echelon (two locations) serial inventory system consisting of a warehouse and a retailer. Customer demands occur only at the retailer according to a Poisson process. The fixed ordering costs are negligible. Thus, we apply the base stock policy (one for one policy) with continuous review in both locations. That is, the retailer makes its orders to the warehouse under $\left(S_{1}-1, S_{1}\right)$ policy, and the warehouse makes its orders to an external supplier with infinite supply under $\left(S_{0}-1, S_{0}\right)$ policy, where $S_{1}$ and $S_{0}$ are the inventory positions in the retailer and in the warehouse, respectively. We consider perishableon-the-shelf items; that is, items have a fixed shelf life 
and start aging on their arrival at the retailer. All transportation times are fixed. All satisfied demands are met based on FIFO (First In First Out) policy. Although the demand not met at the retailer is lost, the unsatisfied demand at the warehouse is backordered. A fixed penalty cost per lost sale and a fixed penalty cost per perished item are incurred at the retailer. Items held in stock both at the warehouse and at the retailers incur holding costs per unit per time unit. Also, the warehouse pays a fixed purchase cost per item. Therefore, shortage, perishing, and holding costs are incurred at the retailer, and purchasing and holding costs are incurred at the warehouse. The objective is to find optimal values of $S_{1}$ and $S_{0}$ to minimize total cost of the system. The following notations are used in the subsequent parts of the paper.

\section{Model Parameters:}

$\mu_{1} \quad$ The customer demand rate;

$\pi \quad$ Cost of a lost sale at the retailer;

$h_{0} \quad$ Holding cost per unit per time unit at the warehouse;

$h_{1} \quad$ Holding cost per unit per time unit at the retailer;

$p \quad$ Cost of a perished item at the retailer;

$c \quad$ Purchase cost per unit at the warehouse;

$\alpha \quad$ The rate of perishing at the retailer;

$\tau_{0} \quad$ Transportation time from the external supplier to the warehouse;

$\tau_{1} \quad$ Transportation time from the warehouse to the retailer;

$m \quad$ The shelf life of the items at the retailer;

$I_{0} \quad$ Average on-hand inventory at the warehouse;

$I_{1} \quad$ Average on-hand inventory at the retailer;

$B_{0} \quad$ The average number of backorders at the warehouse.

\section{Decision variables:}

$S_{0} \quad$ Inventory position at the warehouse;

$S_{1} \quad$ Inventory position at the retailer.

\section{Cost measures:}

$H_{0} \quad$ Average total holding cost per time unit at the warehouse;

$H_{1} \quad$ Average total holding cost per time unit at the retailer;

$\Pi \quad$ Average total shortage cost per time unit at the retailer;
OC Average total perishing cost per time unit at the retailer;

PC Average total purchasing cost per time unit at the warehouse;

$C_{0} \quad$ Total cost rate at the warehouse;

$C_{1} \quad$ Total cost rate at the retailer;

TC Total cost rate of the system.

\section{Cost evaluation}

\subsection{Preliminaries - single installation model}

Schmidt and Nahmias [6] consider a single perishable item, single-location inventory system operating under $(S-1, S)$ policy with lost sales, Poisson demand, outdating costs, purchase costs, and per unit per period holding costs. The results of their model are used to obtain the cost function of the retailer in our model. Therefore, some key results of their model are presented in the following.

Let $X_{k}, 1 \leq k \leq S$, represent the elapsed time since the order of the $k$ th oldest item in the system was placed. That is, $X_{1}$ is the time since the oldest item was ordered, $X_{2}$ is the time since the second oldest item was ordered, etc. Then, the joint density function of $X_{1}, \ldots, X_{S}$ is stated as:

$$
\begin{aligned}
& f_{X_{1}, X_{2}, \ldots, X_{S}}\left(x_{1}, x_{2}, \ldots, x_{S}\right) \\
& = \begin{cases}K e^{-\mu \tau} & \text { for } x_{1}<\tau \\
K e^{-\mu x_{1}} & \text { for } x_{1} \geq \tau\end{cases} \\
& \text { for } x_{1} \geq x_{2} \geq \ldots \geq x_{S} \text { and } S>0,
\end{aligned}
$$

where, $\mu$ and $\tau$ stand for the demand rate and the lead time, respectively. Also, $K$ is a normalizing constant, which is obtained as:

$$
K=\left(\frac{e^{-\mu \tau} \tau^{S}}{S !}+\int_{\tau}^{\tau+m} \frac{x^{S-1} e^{-\mu x}}{(S-1) !} d x\right)^{-1}
$$

Also, using the marginal function of the oldest item, the rate of perishing is obtained (Eq. (7) in [6]):

$$
\alpha=f_{X_{1}}(\tau+m)=\frac{K e^{-\mu(\tau+m)}(\tau+m)^{S-1}}{(S-1) !} .
$$

Furthermore, the proportion of time that the system is out of stock, $P_{0}$, is:

$$
P_{0}=\int_{0}^{\tau} \int_{0}^{x_{1}} \ldots \int_{0}^{x_{S-1}} K e^{-\mu \tau} d x_{S} \ldots d x_{1}=\frac{K e^{-\mu \tau} \tau^{S}}{S !} .
$$

\subsection{Approximate retailer cost}

Since the warehouse may be out of stock when the 
retailer orders an item, the lead time at the retailer is stochastic. That is, the replenishment lead time of the retailer is $\tau_{1}+\Delta$, where $\Delta$ is the delay due to stock-outs at the warehouse. We use the idea of the well-known METRIC model [13] and approximate the lead time by its mean. Thus, the lead time at the retailer is $\tau_{1}^{\prime}=\tau_{1}+\delta$, where $\delta$ is the mean of $\Delta$.

For a given $\tau_{1}^{\prime}$, the retailer's model is quite similar to the single installation model of Schmidt and Nahmias [6]. Therefore, by substitution of $\tau$ and $\mu$ with $\tau_{1}^{\prime}$ and $\mu_{1}$ in Eq. (2), the perishing rate at the retailer is:

$$
\alpha=\frac{K^{\prime} e^{-\mu_{1}\left(\tau_{1}^{\prime}+m\right)}\left(\tau_{1}^{\prime}+m\right)^{S_{1}-1}}{\left(S_{1}-1\right) !},
$$

where from Eq. (1):

$$
K^{\prime}=\left(\frac{e^{-\mu_{1} \tau_{1}^{\prime}} \tau_{1}^{\prime S_{1}}}{S_{1} !}+\int_{\tau_{1}^{\prime}}^{\tau_{1}^{\prime}+m} \frac{x^{S_{1}-1} e^{-\mu_{1} x}}{\left(S_{1}-1\right) !} d x\right)^{-1}
$$

Thus, the perishing cost rate at the retailer is obtained as follows:

$$
\mathrm{OC}=p \alpha=\frac{p K^{\prime} e^{-\mu_{1}\left(\tau_{1}^{\prime}+m\right)}\left(\tau_{1}^{\prime}+m\right)^{S_{1}-1}}{\left(S_{1}-1\right) !} .
$$

Furthermore, the proportion of time that the retailer is out of stock, $P_{0}$, is obtained by substitution of $\tau$ and $\mu$ with $\tau_{1}^{\prime}$ and $\mu_{1}$ in Eq. (3). Thus, $P_{0}$ is:

$$
P_{0}=\frac{K^{\prime} e^{-\mu_{1} \tau_{1}^{\prime}} \tau_{1}^{\prime} S_{1}}{S_{1} !}
$$

Since $P_{0}$ is the proportion of time that the system is out of stock, the proportion of demand that is lost is $\mu_{1} P_{0}$. Therefore, the shortage cost rate at the retailer is:

$$
\Pi=\pi \mu_{1} P_{0}=\pi \mu_{1} \frac{K^{\prime} e^{-\mu_{1} \tau_{1}^{\prime}} \tau_{1}^{\prime S_{1}}}{S_{1} !} .
$$

Let $L$ be the average number of outstanding orders towards the warehouse. Then, by Little's formula, $L=$ $\lambda W$, where $\lambda$ is the order rate and $W$ is the time, an item is on order. In our case, $W=\tau_{1}^{\prime}$ and $\lambda=(1-$ $\left.P_{0}\right) \mu_{1}+\alpha$, which gives $L=\tau_{1}^{\prime}\left(\left(1-P_{0}\right) \mu_{1}+\alpha\right)$. The expected on hand inventory at the retailer is $S_{1}-L$. Thus:

$$
I_{1}=S_{1}-L=S_{1}-\tau_{1}^{\prime}\left(\left(1-P_{0}\right) \mu_{1}+\alpha\right) .
$$

Hence, the holding cost rate at the retailer is:

$$
H_{1}=h_{1} I_{1}=h_{1} S_{1}-h_{1} \tau_{1}^{\prime} \mu_{1}\left(1-P_{0}\right)-h_{1} \tau_{1}^{\prime} \alpha .
$$

Therefore, the total cost rate at the retailer can be obtained from Eqs. (6), (8), and (10) as follows:

$$
\begin{aligned}
C_{1}= & \mathrm{OC}+\Pi+H_{1}=\left(p-h_{1} \tau_{1}^{\prime}\right) \\
& \frac{K^{\prime} e^{-\mu_{1}\left(\tau_{1}^{\prime}+m\right)}\left(\tau_{1}^{\prime}+m\right)^{S_{1}-1}}{\left(S_{1}-1\right) !} \\
& +\left(\pi+h_{1} \tau_{1}^{\prime}\right) \mu_{1} \frac{K^{\prime} e^{-\mu_{1} \tau_{1}^{\prime}} \tau_{1}^{\prime S_{1}}}{S_{1} !}+h_{1} S_{1}-h_{1} \tau_{1}^{\prime} \mu_{1}
\end{aligned}
$$

\subsection{Approximate warehouse cost}

Due to the existence of lost sales and perished items, the retailer demand to the warehouse is not Poisson. Like [12], we ignore this and approximate the demand at the warehouse with a Poisson process with mean $\mu_{0}=\mu_{1}\left(1-P_{0}\right)+\alpha$.

Now, the number of outstanding orders towards the external supplier is as the occupancy level in an $M / D / \infty$ queue. According to Palm's theorem [14], for this type of queues, the steady-state occupancy level is Poisson distributed with mean $\lambda \bar{L}$, where $\lambda$ is the arrival rate and $\bar{L}$ is the mean service time. In our case, $\lambda=\mu_{0}$, and $\bar{L}=\tau_{0}$. Hence, the average on hand inventory and the average number of backorders at the warehouse are obtained as follows:

$$
\begin{aligned}
& I_{0}=\sum_{j=0}^{S_{0}}\left(S_{0}-j\right) \frac{\left(\mu_{0} \tau_{0}\right)^{j}}{j !} e^{-\mu_{0} \tau_{0}}, \\
& B_{0}=\sum_{j=S_{0}+1}^{\infty}\left(j-S_{0}\right) \frac{\left(\mu_{0} \tau_{0}\right)^{j}}{j !} e^{-\mu_{0} \tau_{0}} .
\end{aligned}
$$

Thus, the holding cost rate at the warehouse is:

$$
H_{0}=h_{0} I_{0}=h_{0} \sum_{j=0}^{S_{0}}\left(S_{0}-j\right) \frac{\left(\mu_{0} \tau_{0}\right)^{j}}{j !} e^{-\mu_{0} \tau_{0}} .
$$

Also, the average purchasing cost rate at the warehouse is:

$$
\mathrm{PC}=c \mu_{0}
$$

Therefore, from Eqs. (14) and (15), the warehouse total cost rate is obtained as follows:

$$
C_{0}=\mathrm{PC}+H_{0}=c \mu_{0}+h_{0} \sum_{j=0}^{S_{0}}\left(S_{0}-j\right) \frac{\left(\mu_{0} \tau_{0}\right)^{j}}{j !} e^{-\mu_{0} \tau_{0}}
$$

Furthermore, using Little's formula, the mean of delay at the retailer due to stock-outs at the warehouse, $\delta$, can be obtained as follows:

$$
\delta=\frac{B_{0}}{\mu_{0}}
$$


Therefore, the replenishment lead time of the retailer is approximated as:

$$
\tau_{1}^{\prime}=\tau_{1}+\frac{B_{0}}{\mu_{0}} .
$$

Finally, from Eqs. (11) and (16), total cost rate of the system can be obtained as follows:

$$
\begin{aligned}
\mathrm{TC}= & C_{0}+C_{1}=c \mu_{0}+h_{0} \sum_{j=0}^{S_{0}}\left(S_{0}-j\right) \frac{\left(\mu_{0} \tau_{0}\right)^{j}}{j !} e^{-\mu_{0} \tau_{0}} \\
& +\left(p-h_{1} \tau_{1}^{\prime}\right) \frac{K^{\prime} e^{-\mu_{1}\left(\tau_{1}^{\prime}+m\right)}\left(\tau_{1}^{\prime}+m\right)^{S_{1}-1}}{\left(S_{1}-1\right) !} \\
& +\left(\pi+h_{1} \tau_{1}^{\prime}\right) \mu_{1} \frac{K^{\prime} e^{-\mu_{1} \tau_{1}^{\prime}} \tau_{1}^{\prime} S_{1}}{S_{1} !} \\
& +h_{1} S_{1}-h_{1} \tau_{1}^{\prime} \mu_{1} .
\end{aligned}
$$

\section{Solution procedure}

In Appendix A, we consider a base example and show that Eq. (19) is not convex over $S_{0}$ and $S_{1}$. Therefore, in this section, we present a projection-based solution procedure, named Procedure 1, which constitutes a main loop and an inner loop. The main loop searches over the possible values of $S_{0}$ to find the best one. For each $S_{0}$, an inner loop attempts to determine the best values of $S_{1}$. Evidently, $S_{0}$ and $S_{1}$ cannot be negative for a cost minimizing solution. Also, two lemmas are presented by which we can establish two termination criteria to bound $S_{0}$ and $S_{1}$ from above.

Lemma 1. Given a fixed $\tau_{1}^{\prime}$, the perishing rate at the retailer, $\alpha$, is an increasing function of $S_{1}$. Also $\alpha \rightarrow \infty$ when $S_{1} \rightarrow \infty$.

Proof. See Appendix B.

Lemma 2. For a fixed $\mu_{0}$, total cost rate of the warehouse, $C_{0}$, is a convex function of $S_{0}$.

Proof. This lemma follows from, for example, [15] and [16].

The advantage of Procedure 1 is that with a temporary fixed $S_{0}$, we can use Lemma 1 to establish a termination criterion for the inner loop. In more details, the search over $S_{1}$ in inner loop can be terminated when the perishing cost rate exceeds the best cost found for the retailer, that is when $S_{1}$ satisfies:

$$
\min _{x \leq S_{1}} C_{1}(x) \leq \mathrm{OC}\left(S_{1}\right)=p \alpha .
$$

Furthermore, we can use Lemma 2 to construct a termination criterion for the main loop. Clearly, $C_{0}$ is a lower bound for TC. Therefore, since $C_{0}$ is convex in $S_{0}$, when $S_{0}$ satisfies $\min _{x \leq S_{0}} \mathrm{TC}(x) \leq C_{0}\left(S_{0}\right)$, the search over $S_{0}$ can be terminated.

\section{Procedure 1}

- Step 0: Set $S_{0}:=0, \mathrm{TC}^{\mathrm{min}}:=\pi \mu_{1}, S_{0}^{o p t}:=0$, and $S_{1}^{o p t}:=0$.

- Step 1: Set $s:=0, S_{1}:=1, \tau_{1}^{\prime}:=\tau_{1}, \operatorname{TCS}^{\mathrm{min}}:=$ $\pi \mu_{1}$, and $\mathrm{TCS}^{\mathrm{min}}:=\pi \mu_{1}$. Calculate $\alpha$ from Eq. (4) and $P_{0}$ from Eq. (7).

- Step 2: While TCS1 ${ }^{\text {min }}>p \alpha$, do \{

Calculate $\mu_{0}=\mu_{1}\left(1-P_{0}\right)+\alpha, \tau_{1}^{\prime}$ from Eq. (18) and $C_{1}\left(S_{1}, \tau_{1}^{\prime}\right)$ from Eq. (11).

Calculate $C_{0}\left(S_{0}, \mu_{0}\right)$ from Eq. (16) and set $\mathrm{TC}\left(S_{0}\right):=C_{0}\left(S_{0}, \mu_{0}\right)+C_{1}\left(S_{1}, \tau_{1}^{\prime}\right)$. If $\mathrm{TC}\left(S_{0}\right)<\mathrm{TCS}^{\mathrm{min}}$, then set $\mathrm{TCSO}^{\mathrm{min}}:=\mathrm{TC}\left(S_{0}\right)$, $\mu:=\mu_{0}$, and $s:=S_{1}$.

If $C_{1}\left(S_{1}\right)<\mathrm{TCS}^{\mathrm{min}}$, then set TCS1 ${ }^{\mathrm{min}}:=C_{1}\left(S_{1}\right)$. Set $S_{1}:=S_{1}+1$.

Calculate $\alpha$ from Eq. (4), and $P_{0}$ from Eq. (7). \}

- Step 3: Calculate $C_{0}\left(S_{0}, \mu\right)$ from Eq. (16), If $\mathrm{TCSO}^{\mathrm{min}}<\mathrm{TC}^{\mathrm{min}}$ then $\mathrm{TC}^{\mathrm{min}}:=\mathrm{TCSO}^{\mathrm{min}}$ and let $S_{0}^{o p t}:=S_{0}$ and $S_{1}^{o p t}:=s$.

If $\mathrm{TC}^{\mathrm{min}}<C_{0}\left(S_{0}, \mu\right)$ then set $S^{*}:=\left(S_{0}^{o p t}, S_{1}^{o p t}\right)$, return $S^{*}$ and $\mathrm{TC}^{\mathrm{min}}$, and stop the procedure. Otherwise, set $S_{0}:=S_{0}+1$ and go to Step 1 .

When $S_{0}=S_{1}=0$, all customer demands are lost, which gives total cost of the system equal to $\pi \mu_{1}$. Therefore, in Step 0 and Step 1, we can set all total cost rates to $\pi \mu_{1}$ as a starting point. Step 2 includes the inner loop and search over $S_{1}$. In Step 3, the termination criterion of the main loop is checked and the procedure is continued or stopped, accordingly.

Due to the existence of $\tau_{1}^{\prime}$ and $\mu_{0}$ in cost functions, and their dependence on both $S_{0}$ and $S_{1}$, with the presented termination criteria, Procedure 1 may not obtain the optimal solution which minimizes approximated TC. However, numerical experiments show that in all considered problems this is not the case. In addition, the METRIC-based approximation for the retailer's lead time and Poisson approximation of the warehouse demand are used to obtain Eq. (19). Consequently, the optimal solution based on Eq. (19) is not necessarily the optimal solution of the inventory system. Therefore, apart from Procedure 1, we also propose Procedure 2, which is a neighborhood search based on simulation. In Procedure 2, for a given point $S=\left(S_{0}, S_{1}\right), 8$ neighborhoods including $S=$ $\left(S_{0}-1, S_{1}\right), S=\left(S_{0}+1, S_{1}\right), S=\left(S_{0}, S_{1}-1\right), S=$ $\left(S_{0}, S_{1}+1\right), S=\left(S_{0}-1, S_{1}-1\right), S=\left(S_{0}-1+S_{1}+1\right)$, $S=\left(S_{0}+1, S_{1}-1\right)$, and $S=\left(S_{0}+1, S_{1}+1\right)$ are considered. Then, for each of them, 3 simulations with 
10000 time units are executed. The average cost rate obtained from these 3 simulations is assigned to the corresponding neighborhood. If at least one improving solution has been determined, the neighborhood search is restarted with respect to the best of them. If no superior solution is found, the procedure stops and returns the best found solution. Procedure 2 starts with the obtained solution of Procedure 1.

We conjecture that there is a local optimum, which is also the global optimum. This conjecture is supported by all our numerical tests, yet we cannot formally prove it. Therefore, we consider two termination criteria for Procedure 2; first, if no superior solution is found and, second, if the number of evaluated points exceeds a given upper bound $J_{\max }$. The latter never occurs in our numerical analysis as we set $J_{\max }=50$. Thus, all final solutions are local optimum according to the simulation.

\section{Procedure 2}

- Step 0: Set $j:=1$ and $J_{\max }:=50$. Execute Procedure 1. Let $S^{1}$ be the best policy found by Procedure 1.

- Step 1: Execute 3 simulations with 10000 time units for $S^{j}$ and set $\mathrm{TC}^{j}$ to the average total cost rate obtained by these simulations.

- Step 2: Execute 3 simulations with 10000 time units for all neighborhoods of $S^{j}$, which have not been simulated before. Set $S^{j+1}$ to the policy with the minimum average total cost rate among all neighborhoods. Also, set $T C^{j+1}$ to this total cost rate.
- Step 3: If $\mathrm{TC}^{j+1}<\mathrm{TC}^{j}$ and $j \leq J_{\max }$, set $j:=j+1$ and go to Step 2. Otherwise, return to $S^{j}$ as the best policy and $\mathrm{TC}^{j}$ as its total cost rate. Stop the Procedure.

\section{Numerical analysis}

In this section, a small numerical study is performed to examine effectiveness and accuracy of the proposed procedures. In total, we consider 32 different test problems. We find the best values of $S_{0}$ and $S_{1}$ for each test problem according to Procedures 1 and 2 . Then, effectiveness and accuracy of the procedures are evaluated by simulation. We use a simulation method based on discrete-event simulation. Each simulation consists of 10 runs, each with a run length of 10000 time units. The average cost of the 10 runs is reported as the cost of its corresponding policy. Also, the standard deviation (std) obtained from the 10 runs is presented. The policy with the lowest average simulated cost is introduced as the optimal policy, which is obtained by enumeration over combinations of $S_{0}$ and $S_{1}$. All codes are conducted in MATLAB software.

In Table 1, all combinations of $\mu_{1} \in\{0.5,2\}$, $m \in\{0.5,2\}, p \in\{5,20\}, \pi \in\{20,80\}, c=5, h_{0}=2$, $h_{1}=1$, and $\tau_{0}=\tau_{1}=0.3$ are considered. Also, in Table 2, the results for all combinations of $\tau_{0} \in\{0.3,1\}$, $\tau_{1} \in\{0.3,1\}, p \in\{5,20\}, \pi \in\{20,80\}, c=5, h_{0}=2$, $h_{1}=1$, and $\mu_{1}=m=1$ are presented. The number of problems for which a procedure provides the optimal policy gives an indication of how effective the procedure is when obtaining the optimal policy for the inventory system. For the 32 considered problems, Procedure 1 provides the optimal policy of 21 problems whereas

Table 1. Numerical results for $c=5, h_{0}=2, h_{1}=1$, and $\tau_{0}=\tau_{1}=0.3$.

\begin{tabular}{|c|c|c|c|c|c|c|c|c|c|c|c|c|c|c|}
\hline \multirow[t]{2}{*}{ Problem no. } & \multirow[t]{2}{*}{$\mu_{1}$} & \multirow[t]{2}{*}{$m$} & \multirow[t]{2}{*}{$p$} & \multirow[t]{2}{*}{$\pi$} & \multicolumn{3}{|c|}{ Procedure 1} & \multicolumn{3}{|c|}{ Procedure 2} & \multicolumn{4}{|c|}{ Simulation } \\
\hline & & & & & $S_{0}^{*}$ & $S_{1}^{*}$ & $\mathbf{T C}^{*}$ & $S_{0}^{*}$ & $S_{1}^{*}$ & $\mathrm{TC}^{*}$ & $S_{0}^{*}$ & $S_{1}^{*}$ & $\mathbf{T C}^{*}$ & Std \\
\hline 1 & 0.5 & 0.5 & 5 & 20 & 0 & 0 & 10.00 & 0 & 0 & 10.00 & 0 & 0 & 10.00 & 0 \\
\hline 2 & 0.5 & 0.5 & 5 & 80 & 1 & 1 & 30.86 & 1 & 2 & 27.29 & 1 & 2 & 27.41 & 0.151 \\
\hline 3 & 0.5 & 0.5 & 20 & 20 & 0 & 0 & 10.00 & 0 & 0 & 10.00 & 0 & 0 & 10.00 & 0 \\
\hline 4 & 0.5 & 0.5 & 20 & 80 & 0 & 0 & 40.00 & 0 & 0 & 40.00 & 0 & 0 & 40.00 & 0 \\
\hline 5 & 0.5 & 2 & 5 & 20 & 0 & 1 & 7.57 & 0 & 1 & 7.55 & 0 & 1 & 7.61 & 0.052 \\
\hline 6 & 0.5 & 2 & 5 & 80 & 0 & 2 & 12.12 & 1 & 2 & 12.05 & 1 & 2 & 12.05 & 0.122 \\
\hline 7 & 0.5 & 2 & 20 & 20 & 0 & 0 & 10.00 & 0 & 0 & 10.00 & 0 & 0 & 10.00 & 0 \\
\hline 8 & 0.5 & 2 & 20 & 80 & 1 & 1 & 18.44 & 1 & 1 & 17.79 & 1 & 1 & 18.13 & 0.21 \\
\hline 9 & 2 & 0.5 & 5 & 20 & 0 & 2 & 32.42 & 1 & 2 & 30.15 & 1 & 2 & 30.06 & 0.102 \\
\hline 10 & 2 & 0.5 & 5 & 80 & 2 & 3 & 53.44 & 1 & 3 & 45.31 & 1 & 3 & 45.25 & 0.495 \\
\hline 11 & 2 & 0.5 & 20 & 20 & 0 & 0 & 40.00 & 0 & 0 & 40.00 & 0 & 0 & 40.00 & 0 \\
\hline 12 & 2 & 0.5 & 20 & 80 & 2 & 2 & 83.50 & 1 & 2 & 73.80 & 1 & 2 & 73.01 & 0.434 \\
\hline 13 & 2 & 2 & 5 & 20 & 0 & 3 & 16.59 & 1 & 3 & 16.59 & 0 & 3 & 16.59 & 0.155 \\
\hline 14 & 2 & 2 & 5 & 80 & 1 & 4 & 19.88 & 1 & 4 & 19.69 & 1 & 4 & 19.79 & 0.131 \\
\hline 15 & 2 & 2 & 20 & 20 & 1 & 2 & 19.06 & 1 & 2 & 18.61 & 1 & 2 & 18.42 & 0.126 \\
\hline 16 & 2 & 2 & 20 & 80 & 1 & 3 & 25.01 & 1 & 3 & 24.57 & 1 & 3 & 24.33 & 0.256 \\
\hline
\end{tabular}


Procedure 2 provides the optimal policy of 30 problems. Therefore, Procedure 2 is more effective in terms of obtaining the optimal policy. In fact, this result is what one intuitively would expect, since the obtained policy of Procedure 1 is the start point of Procedure 2 . In Problems 13 and 17, for which Procedure 2 failed to obtain the optimal policy, the cost of the obtained policy lies in $95 \%$ confidence interval of the optimal cost, according to the presented standard deviation. Hence, one can say that Procedure 2 provides the optimal policy for all the considered problems.

Another indication of effectiveness is how much time a procedure needs to obtain the best policy. For each considered problem, in average, Procedure 1 required 0.07 seconds and Procedure 2 required 9.94 seconds. Thus, in terms of the time needed, Procedure 1 is more effective than Procedure 2.

An important aspect of accuracy of the procedures in approximating the cost rate is that the difference in cost rates of a policy for the procedure and for simulation is low. Therefore, the following formula is used to calculate the percentage for the difference of costs, $\% D$.

$$
\% D=\frac{\left|\mathrm{TC}^{P}-\mathrm{TC}^{\text {sim }}\right|}{\mathrm{TC}^{\text {sim }}} \times 100
$$

in which, $\mathrm{TC}^{P}$ is the cost rate obtained using a procedure, whereas $\mathrm{TC}^{\text {sim }}$ is the cost rate obtained from simulation. For each procedure, we only consider the problems for which the policies obtained from the procedure and from simulation are the same. Then, the average of values of $\% D$ for these problems, denoted by $\mathrm{AD}$, is reported as an indicator of the accuracy of the corresponding procedure. A high value of $\mathrm{AD}$ for a procedure means that the procedure is not qualified to approximate the cost rate.

Procedure 1 overestimates the cost rate in 20 out of 21 problems for which it provides optimal policy. Also, the value of AD for Procedure 1 over 21 problems is $3.85 \%$, which means that approximation of Procedure 1 for the cost rate of a policy is in average $3.85 \%$ higher than the real cost rate. The value of AD for Procedure 2 over 30 problems is $0.42 \%$, which means that the estimation of Procedure 2 for the cost rate of a policy is in average $0.42 \%$ different from the real cost rate. Therefore, Procedure 2 is more accurate than Procedure 1 as intuitively was expected.

According to numerical experiments, the approximate model performs rather well. Furthermore, to choose between Procedure 1 and Procedure 2, one should consider the importance of time and accuracy. Form the computational point of view, Procedure 1 outperforms Procedure 2. However, Procedure 2 not only obtains optimal policy for more problems, but also approximates the cost rate more accurately.

\section{Conclusions and future research}

A single perishable item, continuous-review, twoechelon serial inventory system was considered in this paper. Using the well-known METRIC technique, we approximated the stochastic lead time of the retailer with its mean. Also, the demand of the warehouse was approximated as a Poisson process. Then, two procedures were presented for evaluation and optimization of base stock policies. The first procedure was a

Table 2. Numerical results for $c=5, h_{0}=2, h_{1}=1$, and $\mu_{1}=m=1$.

\begin{tabular}{|c|c|c|c|c|c|c|c|c|c|c|c|c|c|c|}
\hline \multirow[t]{2}{*}{ Problem no. } & \multirow[t]{2}{*}{$\tau_{0}$} & \multirow[t]{2}{*}{$\tau_{1}$} & \multirow[t]{2}{*}{$p$} & \multirow[t]{2}{*}{$\pi$} & \multicolumn{3}{|c|}{ Procedure 1} & \multicolumn{3}{|c|}{ Procedure 2} & \multicolumn{4}{|c|}{ Simulation } \\
\hline & & & & & $S_{0}^{*}$ & $S_{1}^{*}$ & $\mathrm{TC}^{*}$ & $S_{0}^{*}$ & $S_{1}^{*}$ & $\mathrm{TC}^{*}$ & $S_{0}^{*}$ & $S_{1}^{*}$ & $\mathrm{TC}^{*}$ & Std \\
\hline 17 & 0.3 & 0.3 & 5 & 20 & 0 & 1 & 15.80 & 0 & 1 & 15.74 & 1 & 1 & 15.78 & 0.123 \\
\hline 18 & 0.3 & 0.3 & 5 & 80 & 1 & 2 & 25.44 & 1 & 2 & 22.33 & 1 & 2 & 22.20 & 0.193 \\
\hline 19 & 0.3 & 0.3 & 20 & 20 & 0 & 0 & 20.00 & 0 & 0 & 20.00 & 0 & 0 & 20.00 & 0 \\
\hline 20 & 0.3 & 0.3 & 20 & 80 & 1 & 2 & 38.81 & 1 & 2 & 35.70 & 1 & 2 & 35.60 & 0.23 \\
\hline 21 & 0.3 & 1 & 5 & 20 & 0 & 2 & 16.65 & 0 & 2 & 16.75 & 0 & 2 & 16.57 & 0.178 \\
\hline 22 & 0.3 & 1 & 5 & 80 & 0 & 5 & 29.07 & 1 & 4 & 25.59 & 1 & 4 & 25.54 & 0.288 \\
\hline 23 & 0.3 & 1 & 20 & 20 & 0 & 0 & 20.00 & 0 & 0 & 20.00 & 0 & 0 & 20.00 & 0 \\
\hline 24 & 0.3 & 1 & 20 & 80 & 1 & 3 & 45.14 & 1 & 3 & 41.57 & 1 & 3 & 41.38 & 0.3 \\
\hline 25 & 1 & 0.3 & 5 & 20 & 0 & 2 & 16.65 & 1 & 1 & 15.72 & 1 & 1 & 15.59 & 0.135 \\
\hline 26 & 1 & 0.3 & 5 & 80 & 2 & 3 & 26.84 & 2 & 3 & 23.06 & 2 & 3 & 23.11 & 0.164 \\
\hline 27 & 1 & 0.3 & 20 & 20 & 0 & 0 & 20.00 & 0 & 0 & 20.00 & 0 & 0 & 20.00 & 0 \\
\hline 28 & 1 & 0.3 & 20 & 80 & 3 & 2 & 40.64 & 2 & 2 & 36.70 & 2 & 2 & 36.95 & 0.32 \\
\hline 29 & 1 & 1 & 5 & 20 & 0 & 2 & 17.14 & 1 & 2 & 15.48 & 1 & 2 & 15.60 & 0.111 \\
\hline 30 & 1 & 1 & 5 & 80 & 2 & 5 & 29.99 & 2 & 4 & 23.40 & 2 & 4 & 23.42 & 0.202 \\
\hline 31 & 1 & 1 & 20 & 20 & 0 & 0 & 20.00 & 0 & 0 & 20.00 & 0 & 0 & 20.00 & 0 \\
\hline 32 & 1 & 1 & 20 & 80 & 2 & 3 & 46.16 & 2 & 3 & 38.75 & 2 & 3 & 38.56 & 0.362 \\
\hline
\end{tabular}


heuristic based on the approximated model and, from a computational point of view, it was very efficient and simple. The second procedure was a simulation-based neighborhood search which started from the obtained solution of the first procedure and modified the quality of the solution. Therefore, the second procedure was more accurate than the first one. Numerical results indicate that the proposed procedures perform rather well.

To the best of our knowledge, no paper is yet published which deals with both perishability and lost sales in a continuous-review multi-echelon inventory setting. This paper presents an approach to consider this problem. Although a serial system is considered, the presented model could be easily extended to a distribution system with identical retailers.

A valuable work for future research is to develop a model for lost sale case when items start perishing at the warehouse. Such a model is more complex than ours, since the retailer deals with items with stochastic shelf life. Considering the ordering cost is also important and would be a challenge for future research.

\section{References}

1. Abdel-Malek, L. and Ziegler, H. "Age dependent perishability in two-echelon serial inventory systems", Comput. Oper. Res., 15, pp. 227-238 (1988).

2. Fujiwara, O., Soewandi, H. and Sedarage, D. "An optimal ordering and issuing policy for a two stage inventory system for perishable products", Eur. J. Oper. Res., 99, pp. 412-424 (1997).

3. Kanchanasuntorn, K. and Techanitisawad, A. "An approximate periodic model for fixed-life perishable products in a two-echelon inventory-distribution system", Int. J. Prod. Econ., 100, pp. 101-115 (2006).

4. Olsson, F. "Modeling two-echelon serial inventory systems with perishable items", IMA J. Manage. Math., 21, pp. 1-17 (2010).

5. Mahmoodi, A. and Haji, A. " $(1, T)$ policy for a twoechelon inventory system with perishable-on-the-shelf items", J. Opt. Ind. Eng., 16, pp. 31-40 (2014).

6. Schmidt, C.P. and Nahmias, S. " $(S-1, S)$ policies for perishable inventory", Manage. Sci., 31, pp. 719-728 (1985).

7. Perry, D. and Posner, M. "An $(S-1, S)$ inventory system with fixed shelflife and constant lead times", Oper. Res., 46, pp. 65-71 (1998).

8. Olsson, F. and Tydesjo, P. "Inventory Problems with perishable items: Fixed lifetime and backlogging", Eur. J. Oper. Res., 202, pp. 131-137 (2010).

9. Mahmoodi, A., Haji, A. and Haji, R. "One for one period policy for perishable inventory", Comp. Ind. Eng., 79, pp. 10-17 (2015).
10. Nahmias, S. "Perishable inventory theory: a review", Oper. Res., 30, pp. 680-708 (1982).

11. Karaesman, I., Scheller-Wolf, A. and Deniz, B. "Managing perishable and aging inventories: Review and future research directions", In Planning Production and Inventories in the Extended Enterprise, K. Kempf, P. Keskinocak, and P. Uzsoy, Eds., Springer, US, pp. 393-436 (2011)

12. Andersson, J. and Melchiors, P. "A two-echelon inventory model with lost sales", Int. J. Prod. Econ., 69, pp. 307-315 (2001).

13. Sherbrooke, C.C. "METRIC: A multi-echelon technique for recoverable item control", Oper. Res., 16, pp. 122-141 (1968).

14. Palm, C. "Analysis of the Erlang traffic formula for busy signal assignment", Ericson Technics, 5, pp. 3958 (1938).

15. Axsater, S. "Continuous review policies for multi-level inventory systems with stochastic demand", In Handbooks in $O R$ and $M S$, S.C. Graves, A.H.G. Rinnooy Kan, and P. Zipkin, Eds., 4th Edn., pp. 175-197, North-Holland, Amsterdam (1993).

16. Zipkin, P., Foundations of Inventory Management, McGraw Hill, Boston (2000).

\section{Appendix A}

\section{Convexity of Eq. (19)}

To investigate the convexity of the approximated total cost function in Eq. (19), we consider a basic case with the following parameters:

$$
\begin{aligned}
& c=5, h_{0}=2, h_{1}=1, \\
& \tau_{0}=\tau_{1}=0.3, \mu_{1}=2, \\
& m=2, p=5, \text { and } \pi=20 .
\end{aligned}
$$

For this example, a 3 -dimensional graph of $\mathrm{TC}\left(S_{0} ; S_{1}\right)$ (Eq. (19)) as a function of the two variables $S_{0}$ and $S_{1}$ is provided using Matlab Software. As can be seen in Figure A.1, $\mathrm{TC}\left(S_{0} ; S_{1}\right)$ is not convex.

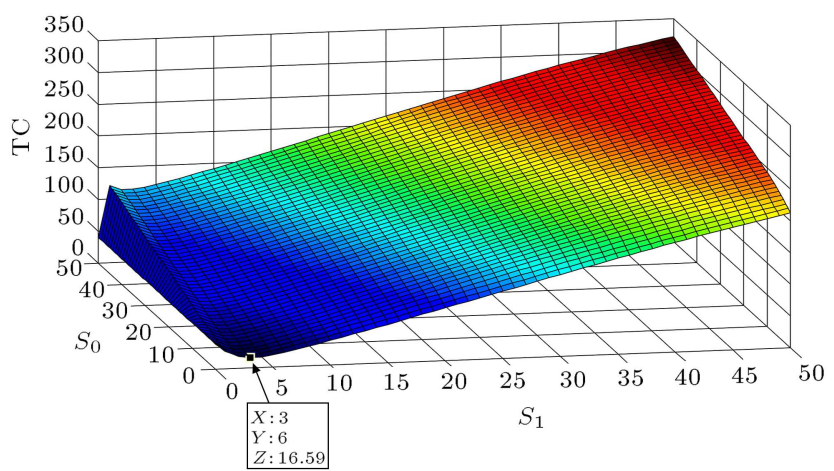

Figure A.1. The graph of $\operatorname{TC}\left(S_{0} ; S_{1}\right)$ for the basic example. 


$$
\begin{aligned}
& \frac{\alpha\left(S_{1}+1\right)}{\alpha\left(S_{1}\right)}=\frac{e^{-\mu_{1}\left(\tau_{1}^{\prime}+m\right)}\left(\tau_{1}+m\right)^{S_{1}} /\left(\left\{\frac{e^{-\mu_{1} \tau_{1}^{\prime} \tau_{1}^{\prime} S_{1}+1}}{\left(S_{1}+1\right) !}+\int_{\tau_{1}^{\prime}}^{\tau_{1}^{\prime}+m} \frac{x^{S_{1}} e^{-\mu_{1} x}}{S_{1} !} d x\right\} S_{1} !\right)}{e^{-\mu_{1}\left(\tau_{1}^{\prime}+m\right)}\left(\tau_{1}^{\prime}+m\right)^{S_{1}-1} /\left(\left\{\frac{e^{-\mu_{1} \tau_{1}^{\prime}} \tau_{1}^{\prime} S_{1}}{S_{1} !}+\int_{\tau_{1}^{\prime}}^{\tau_{1}^{\prime}+m} \frac{x^{S_{1}-1} e^{-\mu_{1} x}}{\left(S_{1}-1\right) !} d x\right\}\left(S_{1}-1\right) !\right)} \\
& =\frac{\left(\tau_{1}^{\prime}+m\right)\left\{\left(e^{-\mu_{1} \tau_{1}^{\prime}} \tau_{1}^{\prime S_{1}} / S_{1}\right)+\int_{\tau_{1}^{\prime}}^{\tau_{1}^{\prime}+m} x^{S_{1}-1} e^{-\mu_{1} x} d x\right\}}{\left(e^{-\mu_{1} \tau_{1}^{\prime}} \tau_{1}^{\prime S_{1}+1} /\left(S_{1}+1\right)\right)+\int_{\tau_{1}^{\prime}}^{\tau_{1}^{\prime}+m} x^{S_{1}} e^{-\mu_{1} x} d x}
\end{aligned}
$$

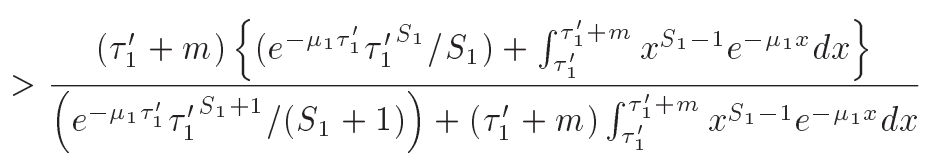

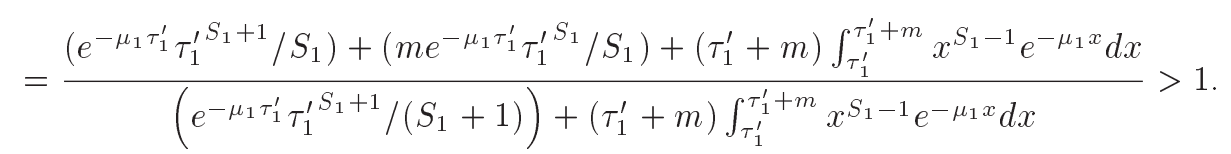

\section{Box I}

$$
\begin{aligned}
& \alpha\left(S_{1}\right)=\frac{e^{-\mu_{1}\left(\tau_{1}^{\prime}+m\right)}\left(\tau_{1}^{\prime}+m\right)^{S_{1}-1}}{\left\{\frac{e^{-\mu_{1} \tau_{1}^{\prime} \tau_{1}^{\prime} S_{1}}}{S_{1} !}+\int_{\tau_{1}^{\prime}}^{\tau_{1}^{\prime}+m} \frac{x^{S_{1}-1} e^{-\mu_{1} x}}{\left(S_{1}-1\right) !} d x\right\}\left(S_{1}-1\right) !}=\frac{e^{-\mu_{1}\left(\tau_{1}^{\prime}+m\right)}}{\frac{e^{-\mu \tau_{1}^{\prime} \tau_{1}^{\prime} S_{1}}}{S_{1}\left(\tau_{1}^{\prime}+m\right)^{S_{1}-1}}+\int_{\tau_{1}^{\prime}}^{\tau^{\prime}+m}\left(\frac{x}{\tau_{1}^{\prime}+m}\right)^{S_{1}-1} e^{-\mu_{1} x} d x} \\
& >\frac{e^{-\mu_{1}\left(\tau_{1}^{\prime}+m\right)}}{\frac{e^{-\mu \tau_{1}^{\prime} \tau_{1}^{\prime} S_{1}}}{S_{1}\left(\tau_{1}^{\prime}+m\right)^{S_{1}-1}}+\int_{\tau_{1}^{\prime}}^{\tau_{1}^{\prime}+m}\left(\frac{x}{\tau_{1}^{\prime}+m}\right)^{S_{1}-1} d x}=\frac{e^{-\mu_{1}\left(\tau_{1}^{\prime}+m\right)}}{\frac{e^{-\mu_{1} \tau_{1}^{\prime}} \tau_{1}^{\prime}}{S_{1}}\left(\frac{\tau^{\prime}}{\tau_{1}^{\prime}+m}\right)^{S_{1}-1}+\frac{\left(\tau_{1}^{\prime}+m\right)^{S_{1}}}{S_{1}\left(\tau_{1}^{\prime}+m\right)^{S_{1}-1}}-\frac{\tau_{1}^{\prime} S_{1}}{S_{1}\left(\tau_{1}^{\prime}+m\right)^{S_{1}-1}}} \\
& =\frac{S_{1} e^{-\mu_{1}\left(\tau_{1}^{\prime}+m\right)}}{e^{-\mu_{1} \tau_{1}^{\prime}} \tau_{1}^{\prime}\left(\frac{\tau^{\prime}}{\tau_{1}^{\prime}+m}\right)^{S_{1}-1}+\tau_{1}^{\prime}+m-\tau_{1}^{\prime}\left(\frac{\tau^{\prime}}{\tau_{1}^{\prime}+m}\right)^{S_{1}-1}} .
\end{aligned}
$$

Box II

\section{Appendix B}

\section{Proof of Lemma 1}

First, we show that $\alpha\left(S_{1}\right)$ is increasing in $S_{1}$, which is same as showing that $\frac{\alpha\left(S_{1}+1\right)}{\alpha\left(S_{1}\right)}>1$. From Eqs. (4) and (5), the equation shown in Box I is obtained. Furthermore, for the second part, the equation shown in Box II is obtained, where:

$$
\lim _{S_{1} \rightarrow \infty} \frac{S_{1} e^{-\mu_{1}\left(\tau_{1}^{\prime}+m\right)}}{e^{-\mu_{1} \tau_{1}^{\prime}} \tau_{1}^{\prime}\left(\frac{\tau_{1}^{\prime}}{\tau_{1}^{\prime}+m}\right)+\tau_{1}^{\prime}+m-\tau_{1}^{\prime}\left(\frac{\tau^{\prime}}{\tau_{1}^{\prime}+m}\right)^{S_{1}-1}}=\infty
$$

\section{Biographies}

Anwar Mahmoodi is currently a $\mathrm{PhD}$ candidate in Industrial Engineering at Sharif University of Technology. He received his BSc in Industrial Engineering from
Isfahan University of Technology and his MSc degree from Sharif University of Technology. His research interests include supply chain management, inventory control, and queuing theory.

Alireza Haji is currently an Associate Professor of Industrial Engineering at Sharif University of Technology, Tehran, Iran. He received his BSc, MSc, and $\mathrm{PhD}$ degrees in Industrial Engineering from Sharif University of Technology, Tehran, Iran, in 1987, 1991, and 2001, respectively. His research interests include inventory control, project control, project management, stochastic models, and queuing systems.

Rasoul Haji is currently a Professor of Industrial Engineering at Sharif University of Technology in Tehran, Iran. He received a BSc degree from University 
of Tehran in Chemical Engineering, in 1964. In 1967, he received his MSc degree and then, in 1970, his $\mathrm{PhD}$ degree both in Industrial Engineering from the University of California-Berkeley. He is recognized as a co-founder of a fundamental and important relation in queuing theory, known as "Distributional Little's Law". His research interests include inventory control, stochastic processes, and queuing theory. 